\title{
Gene analysis of seven cases of primary immunodeficiency
}

\author{
Ying Zhu, Li Li, Guoshun Mao, Lei Zhang, Jing Wang, Nannan Li \\ Department of Pediatrics, Fuyang City People's Hospital, Fuyang 236000, China \\ Contributions: (I) Conception and design: Y Zhu; (II) Administrative support: G Mao; (III) Provision of study materials or patients: L Li, J Wang; \\ (IV) Collection and assembly of data: N Li; (V) Data analysis and interpretation: Y Zhu, L Zhang; (VI) Manuscript writing: All authors; (VII) Final \\ approval of manuscript: All authors. \\ Correspondence to: Ying Zhu. Department of Pediatrics, Fuyang City People's Hospital, 63 Luci street, Yingzhou District, Fuyang 236000, China. \\ Email: zhuyingz163@163.com.
}

Background: Primary immune deficiency diseases (PID) are a group of potentially serious disorders in
which inherited defects in the immune system lead to increased infections. This paper explores the clinical
characteristics and pathogenic gene mutation of PID.
Methods: The clinical data, clinical manifestations, and gene sequencing results of seven children were analyzed.

Results: Among the seven children, six were male, and one was female, aged from 4 months to 13 years old. All of them had a history of repeated infection and pneumonia. High throughput sequencing (NGS) showed that the BTK gene of case 1 had c.1921c > t mutation; the BTK gene of case 2 had c.906-908del splice site mutation; the BTK gene of case 3 had c.718delg mutation; the cybb gene of case 4 had c.469c $>$ t mutation; the IL2RG gene of case 5 had c.202g > A mutation; the STAT1 gene of case 6 had c.854a > G mutation; the case 7 had c.718delg mutation. There was c. $1154 \mathrm{c}>\mathrm{t}$ mutation in the STAT1 gene. Cases 1, 3, 6 and 7 were new mutations, and cases 2, 4, and 5 were inherited from mothers.

Conclusions: In clinical cases of children with recurrent infection, the immunologic index is abnormal, so we need to be highly aware of the possibility of PID, and timely high-throughput sequencing is helpful for the diagnosis.

Keywords: Primary immunodeficiency; gene mutation; clinical characteristics; drug effect

Submitted Feb 19, 2020. Accepted for publication Mar 05, 2020.

doi: $10.21037 /$ tp.2020.03.07

View this article at: http://dx.doi.org/10.21037/tp.2020.03.07

\section{Introduction}

Primary immune deficiency diseases (PID), also known as congenital immune deficiency diseases, are diseases caused by immune dysfunction due to genetic abnormalities of the immune system or congenital immune system development disorders, including primary B-cell defects, primary T-cell defects, and primary combined immunodeficiency, and most of them are single-gene genetic diseases (1-3). At present, more than 350 kinds of PID related gene defects have been found, which may lead to multiple clinical manifestations including repeated infection or opportunistic bacterial infection, autoimmune and malignant tumor (4). With the combination of basic research and clinical medicine, potential immunological mechanism and genetic defects in PID have been found gradually, which provides essential support for the diagnosis, classification, and treatment of diseases, and high-throughput sequencing (NGS) technology provides an essential means for the diagnosis of such diseases (5). In this study, we used NGS to sequence the genes of suspected primary immunodeficiency patients and their immediate relatives, to help diagnose and provide 
genetic counseling, and to deepen the understanding of PID further.

\section{Methods}

\section{Research object}

From May 2017 to August 2018, 7 children with PID who were hospitalized and diagnosed in pediatrics of Fuyang People's Hospital were selected as the study object. All cases showed repeated infection, immunoglobulin, and lymphocyte subsets were abnormal in varying degrees, which need to be confirmed at the molecular genetic level by the NGS method.

\section{Research methods}

Collect the clinical data of patients, including clinical manifestations, laboratory tests, etc. $2 \mathrm{~mL}$ peripheral venous blood of proband and lineal family were collected, and the pathogenic genes were detected by the method of NGS, but 2 of them were detected in the hospital. The family members have approved this study of the children and the medical ethics committee of the hospital.

\section{Results}

Among the seven children, six were male, and one was female, aged 4 months, 1 year, 1 year, 9 months, 7 years, 10 years, and 13 years. Among them, 5 were $\mathrm{X}$-linked genetic diseases [including $3 \mathrm{X}$-linked agammaglobulinemias (XLA), $1 \mathrm{X}$-linked chronic granulomatous disease (xcgd), 1 $\mathrm{X}$-linked severe immunodeficiency disease ( $\mathrm{x}$-linked severe com)]. Two cases of PID (STAT1 defect). All the parents of the patients were not close relatives, and their mothers were healthy during pregnancy, with 6 cases of full-term delivery and 1 case of premature delivery. The clinical data, laboratory examination, and gene test results of each case are shown in Table 1.

\section{Clinical data}

All the seven children had repeated high fever, which showed different degrees and distinct parts of the infection. Pulmonary infection was the most common. In addition to pulmonary infection, 2 cases were characterized by digestive system symptoms ( 1 case with recurrent diarrhea, 1 case with chronic superficial gastritis, hepatobiliary dilatation, etc.); 1 case with nervous system symptoms; 1 case with severe sepsis and multiple organ dysfunction.

\section{Laboratory test results}

In case 1 , case 2 and case 3 , the immunoglobulin was significantly decreased without the decrease of lymphocyte subsets; in case 4, the immunoglobulin and lymphocyte subsets were not significantly abnormal; in case 5 and case 7 , the immunoglobulin and lymphocyte subsets were significantly decreased, which was consistent with the severity of the clinical manifestations of the children; in case 6 , the lymphocyte subsets were significantly decreased, and the immunoglobulin was not abnormal.

\section{Gene test results}

The mutation of c.1921c $>t$ was found in exon 19 of the BTK gene in case 1 by NGS. The mutation resulted in the mutation of amino acid of protein 641 encoded by the gene from arginine to cysteine (p. arg641cys). The mutation was not detected in the family sequencing data. Case 2 had a deletion mutation (c.906-908del) in the BTK gene, which resulted in the deletion of glycine at 303 (p.gly303x) encoded by the gene, which was inherited from the mother of the child. The mutation of c.718delg was found in exon 8 of the BTK gene in case 3. The mutation resulted in a code shift mutation (p. glu240fs) at the 240 positions of the protein encoded by the gene. The mutation was not detected in the family sequencing data. In case 4, exon 5 of cybb gene had c.469c > t mutation, which resulted in the mutation of 157 amino acids of cybb protein from arginine to termination codon $(\mathrm{p} \cdot \arg 15 \mathrm{x})$, resulting in a truncated protein, which was inherited from the mother of the child. The mutation of c.202g > A in exon 2 of the IL2RG gene in case 5 resulted in the mutation of 68 amino acids of the protein encoded by the gene from glutamate to lysine (p.glu68lys), which originated from the mother of the child. In case 6 , the mutation of c.854a $>\mathrm{G}$ was found in exon 10 of the STAT1 gene, which resulted in the mutation of 285 amino acids of the protein encoded by the gene from glutamic acid to aspartic acid (p.glu285asp). The mutation of the disease was not detected in the family sequencing data. In Example 7, c.1154c > t mutation was found in the STAT1 gene, which caused the amino acid at 385 positions of the protein encoded by the gene to change from threonine to methionine (p.thr385met). The sequencing 


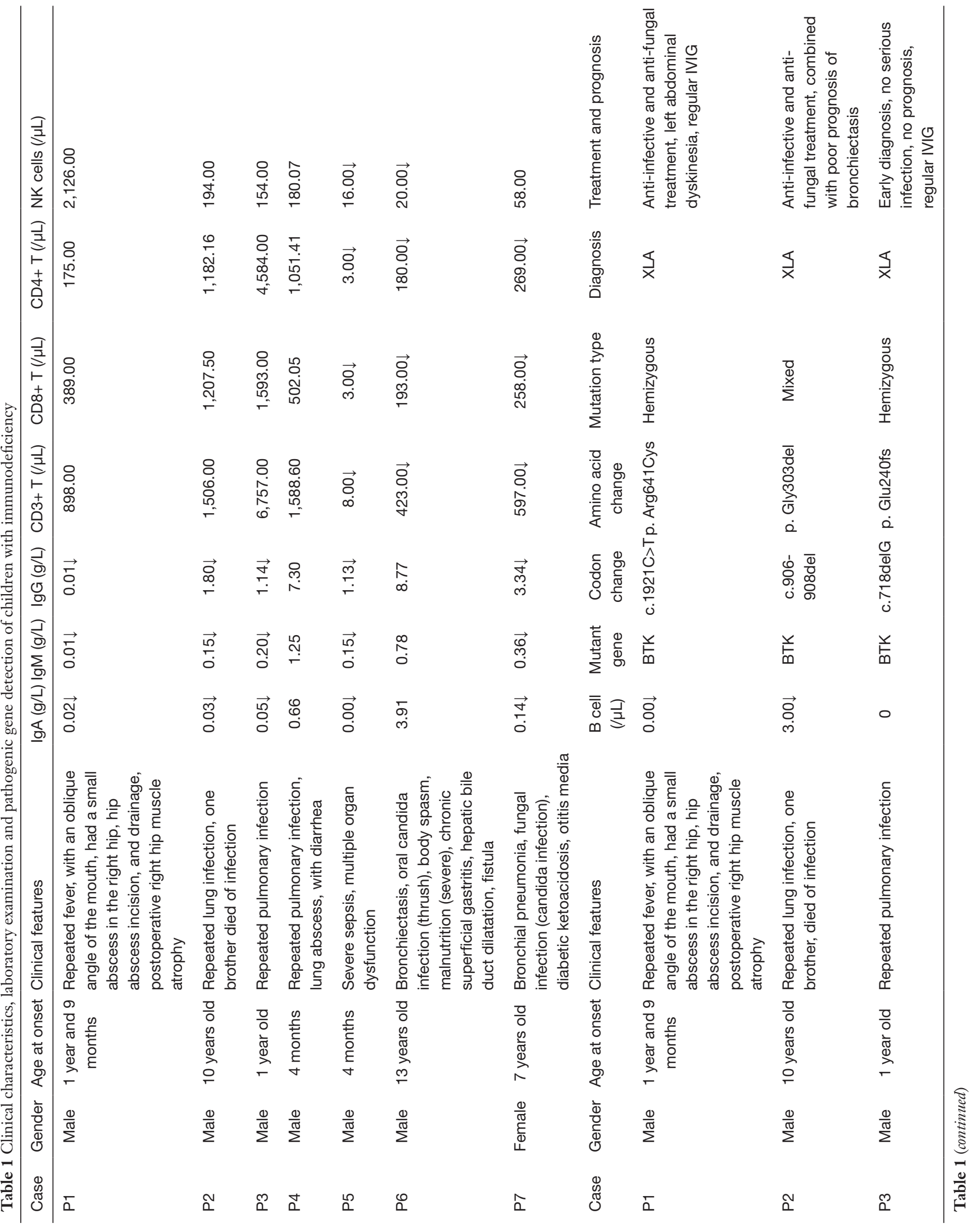


results of NGS are shown in Figure 1.

\section{Treatment and follow-up}

Case 1, case 2 and case 3 were diagnosed as XLA, and case 1 was treated with intravenous immunoglobulin regularly, leaving half limb movement disorder; case 2 improved after anti-infection and antifungal treatment, but complicated with bronchiectasis, with poor prognosis; case 3 was diagnosed earlier due to the high vigilance of our doctors, with a better prognosis in the case of uncompleted and severe infection, and now was treated with intravenous immunoglobulin regularly. Case 4 was diagnosed as xcgd. Infection control after anti-bacteria and anti-fungus treatment were conducted. Hematopoietic stem cell transplantation was conducted in Shanghai. At present, the general situation is $\mathrm{OK}$, and regular follow-up treatment is in progress. Case 5 was diagnosed as XSCID, accompanied by severe sepsis and multiple organ dysfunction. The patient was admitted to the hospital for anti-infection and support treatment and died after giving up treatment. Case 6 was diagnosed as an immunodeficient disease (STAT1 deficiency) and was treated with anti-infective and antifungal therapy after admission. Case 7 was diagnosed as immune deficiency disease (STAT1 deficiency) and type I diabetes and was treated with anti-infective and antifungal therapy after admission, standardized management of diabetes, and follow-up treatment after discharge.

\section{Discussion}

PID is a rare genetic disease. The incidence of PID in the United States is about 1/1,200 live births. At present, there are different reports in China, but the diagnosis rate is less than $5 \%(6)$. Since the discovery of X-linked agammaglobulinemia in 1952, more than 350 gene defects have been reported to cause PID (7). In addition to the diseases as dangerous as SCID, the diseases also include the susceptibility to opportunistic infection, persistent inflammation, and severe organ-specific autoimmune diseases. Without prompt diagnosis and treatment, it may lead to chronic, dangerous, and often life-threatening infection and/or life-threatening autoimmune diseases. However, due to the wide variety of PID and the lack of specificity, clinical diagnosis is difficult (8). With the development of gene diagnosis technology, highthroughput sequencing technology plays a vital role in the early diagnosis and treatment of PID. In 2011, based on 
P1
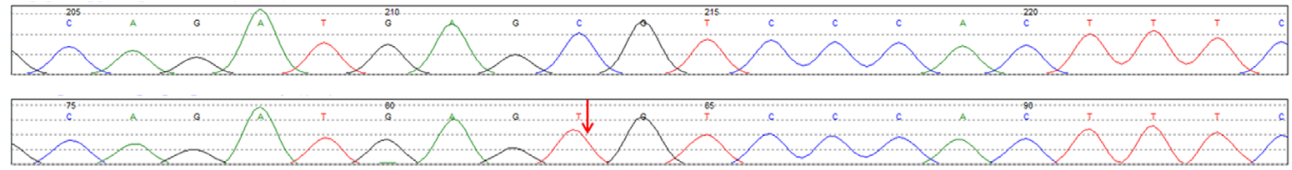

BTK (Xq21|NM_000061.2) Exon19 c.1921C>T p. (Arg641Cys)

P2
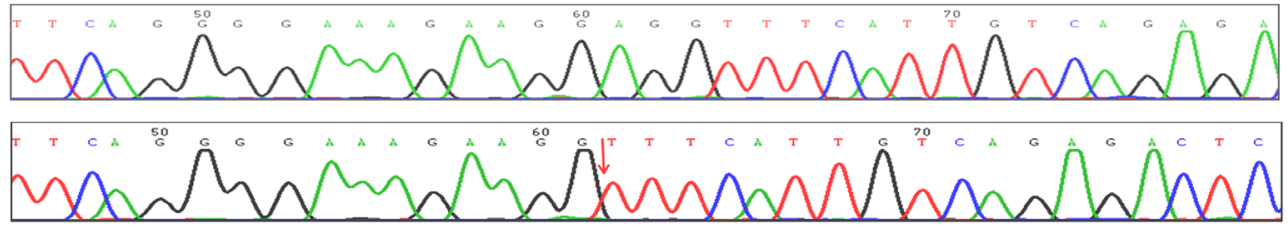

BTK (Xq21|NM_000061.2) Exon19. c.906-908del p.(Gly303del)

P3

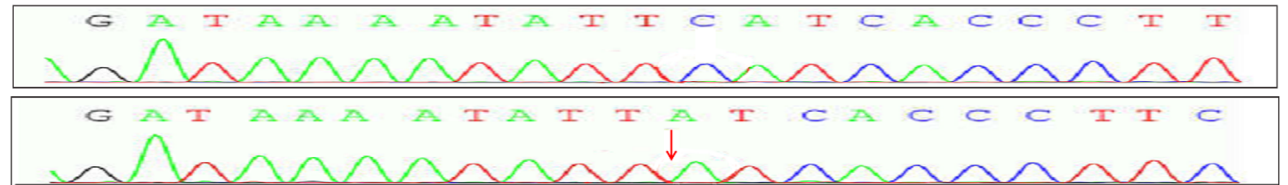

BTK (Xq21|NM_000061.2) Exon8 c.718delG p.(Glu240fs)

P4

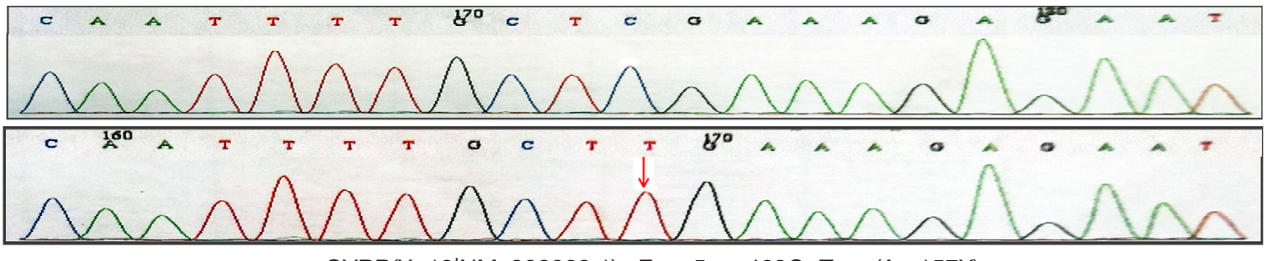

CYBB(Xp13|NM_000023.1) Exon5 c.469C >T p.(Arg157X)

P5

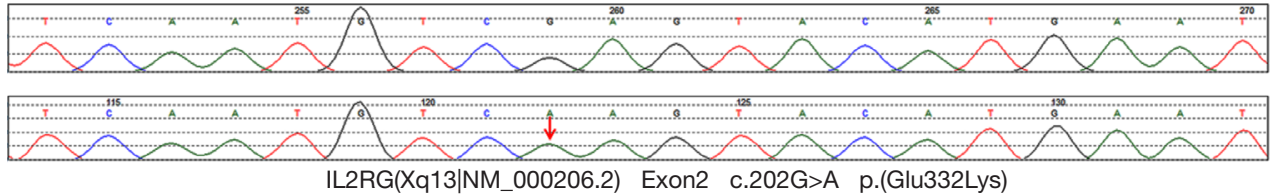

P7

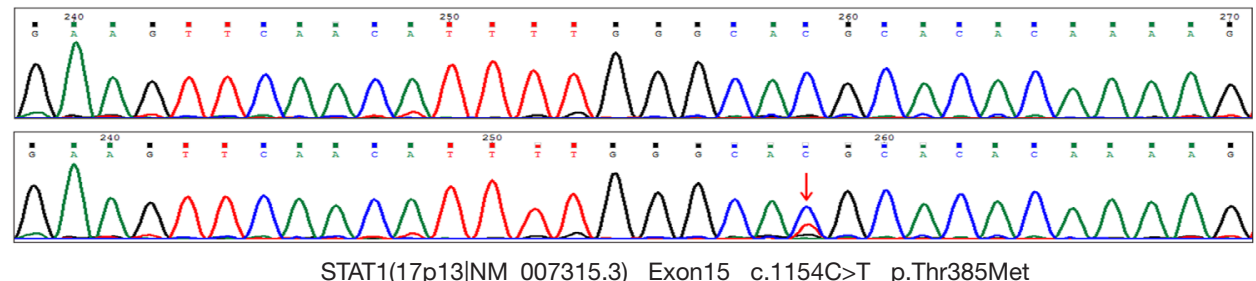

Figure 1 The BTK gene of case 1 has c.1921c > t mutation; the BTK gene of case 2 has c.906-908del splicing site mutation; the BTK gene of case 3 has c.718delg mutation; the cybb gene of case 4 has c.469c > t mutation; the IL2RG gene of case 5 has c.202g > mutation; the STAT1 gene of case 7 has c. $1154 \mathrm{c}>\mathrm{t}$ mutation. The arrow shows the mutation site. The STAT1 gene of case 6 had c.854a $>$ G mutation, and the sequencing map was not obtained (the parents only provided the report sheet, and the sequencing map was lost).

modern gene diagnosis technology, the latest classification standard of Expert Committee of International Federation of immunology divides PID into eight categories: (I) immune deficiency with abnormal cellular and/or humoral immunity; (II) combined immune deficiency; (III) immune deficiency with antibody deficiency; (IV) immune regulation imbalance disease; (V) number and/or function defect of congenital phagocytes; (VI) innate immunity. Disease 
defect; (VII) self-inflammatory disease; (VIII) complement defect (9).

XLA is one of the most common types of primary defects, also known as Bruton's disease, which is X-linked recessive inheritance. It is mostly seen in male infants and young children, and it is manifested as the decrease or deletion of $\mathrm{B}$ cell number in blood circulation and lymphoid tissue, the apparent decrease or deletion of various immunoglobulin levels in serum, and the average number and function of $T$ cell (10-12). It is found that the pathogenic gene of XLA is the Brunton's tyrosine kinase (Btk) gene. The defect of this gene can cause the synthesis obstacle of tyrosine kinase. The development of $B$ cells stops in the state of pro-B cells, resulting in the decrease or even deletion of mature B cells (13). At present, more than 800 mutations have been found in Btk, including missense mutation, nonsense mutation, insertion/deletion mutation, and splice site mutation $(14,15)$. In this study, the level of immunoglobulin, and the number of B lymphocytes in peripheral blood of case 1, case 2 and case 3 were significantly reduced, which was consistent with the development of XLA. Sequencing results showed that there were c.1921c > t-missense mutation, c.906908del deletion mutation and c.718delg mutation in the Btk gene of three children, respectively. These mutation types were known as XLA pathogenic variation, which could be diagnosed as XLA. However, case 1 and case 3 of family sequencing data did not detect the mutation gene carried by the mother, which is inconsistent with the literature report. The new mutation of the child comes from the parents' gonad chimerism (sperm or egg) or spontaneous mutation during the development of the fertilized egg, which may be related to the external stimulation such as diet, emotion, radioactive material contact history during pregnancy, but the reason for the new mutation is still unknownindefinite determinism. According to the literature reports, the $c .1921 \mathrm{c}>\mathrm{t}$ missense mutation in case 1 is located in the protein kinase domain, causing p.arg641cys, Kobayashi and Jin h reported two cases of XLA caused by c.arg1085 > a missense mutation $(16,17)$, and bioinformatics predicted that the $c .1921 \mathrm{c}>\mathrm{t}$ mutation might cause disease so that the mutation can be classified as "suspected pathogenic" mutation. In case 2, the c.906-908del deletion mutation predicted by Alamut function software may affect the function of the protein domain. Gaspar HB also reported that the mutation might lead to "gamma globulin deficiency", which is classified as "suspected pathogenic" mutation (18).

Chronic granulomatous disease (CGD) is a hereditary primary immunodeficiency disease caused by functional damage of NADPH oxidase complex in neutrophils and monocytes (19). NADPH oxidase complex is composed of membrane-binding proteins and cytoplasmic proteins. When phagocytes are activated, these proteins cooperate to produce ROS, which participates in the ordinary killing of bacteria/fungi. The transmembrane glycoprotein gp91phox is encoded by cybb on the $\mathrm{X}$ chromosome. It is a vital part protein in the NADPH oxidase complex. The CGD cases caused by mutations account for about two-thirds of the total $(20,21)$. The incidence in the United States is (200,000-250,000), but it is not clear in China (22). Xcgd is common in men. Except for women with $\mathrm{X}$-chromosome inactivation related diseases, women carriers have no clinical symptoms (23). Repeated and severe infection is the prominent manifestation of this disease. Pulmonary lesions are common, and immunoglobulin and lymphocyte classification are often standard. This test can be used to distinguish from other primary immunodeficiency diseases such as severe joint immunodeficiency, XLA, high IgM syndrome, and high IgE syndrome. In this study, case 4 was diagnosed as "pneumonia and pulmonary abscess of the newborn due to the hospitalization for shortness of breath for 2 weeks". After discharge, there were repeated infections. The clinical symptoms and immunological tests were consistent with xcgd. At the age of 4 months, the gene sequencing c. $469 c>t$ mutation resulted in the nonsense mutation of amino acid. The mutation did not belong to the polymorphism site, and the frequency was very infrequent in the population. Ariga et al. Reported that the mutation was related to CGD (24), Ko et al. also reported a nonsense mutation caused by c.469c > t (25) in 1996, so the site was determined as a pathogenic mutation.

STAT1 is a cytoplasmic protein involved in signal transduction from type I IFN (IFN- $\alpha$ and IFN- $\beta$ ) and IFN- $\gamma$. STAT1 gene mutation can cause autosomal recessive, autosomal dominant, autosomal dominant, autosomal dominant, autosomal dominant, and autosomal dominant Unofficially 31C, Mycobacterium, autosomal dominant (26). STAT protein is a family of transcription factors that mediate cellular responses to cytokines and growth factors. It was found that mutations of four STAT family genes can cause autoimmune diseases. Homozygous or complex heterozygous mutations of STAT1 result in complete or partial deletion of STAT1 protein, which 
is related to the susceptibility of intracellular pathogens to herpes infection. Chronic mucocutaneous candidiasis (CMC) is common in patients with heterozygous STAT1 function acquisition (GoF) mutations, but it can also be associated with a bacterial and viral infection, autoimmune manifestations, lymphopenia, cerebral aneurysm, and increased risk of tumor. Stat 2 deficiency is related to the selectivity and susceptibility of virus infection, while the loss of function (LOF) mutation of STAT3 can lead to autosomal dominant, hyper IgE syndrome (HIES). Lof mutation and STAT3 GOF mutation of STAT5b are related to diseases characterized by autoimmune or allergic manifestations, with an increased risk of infection. In particular, STAT5b deficiency leads to growth hormone (GH) insensitivity, immune deficiency, diarrhea, and generalized eczema, while STAT3 GOF mutation leads to autoimmune hemocytopenia, lymphadenopathy, dwarfism, infection, enteropathy and multiple organ autoimmunity (27). STAT1 heterozygous variation was detected in case 6 of this study. The clinical manifestations were recurrent upper and lower respiratory tract infection, recurrent thrush, bronchiectasis since the age of 3 months (13 years old), consistent with the characteristics of this disease. However, no parents carrying this variation was detected in the family, which may be a "new" variation. However, the mutation of c. $1145 \mathrm{c}>\mathrm{t}$ in STAT1 detected in case 7 has been reported to cause immune deficiency disease, which can be classified as pathogenic mutation. However, in the past, this case of a child caused CMC due to STAT1; although there is immune deficiency compared with this child, the clinical symptoms are not entirely consistent. Moreover, this case of a child with type I diabetes mellitus has not been reported to directly cause I by STAT1 mutation. The relationship between type I diabetes and STAT1 mutation needs further study (28).

Severe combined immune deficiency (SCID) is a group of inherited diseases with thymus and lymphoid tissue hypoplasia and $\operatorname{IgE}$ deficiency. The mechanism is that it cannot produce humoral and cellular immune responses (29). A variety of gene defects can cause it. Interleukin-2 receptor (IL2RG) gene is an essential pathogenic gene. When the $\gamma$ chain gene of IL2RG mutates, the development of T cells lags behind the pro-T stage, and the development of $\mathrm{B}$ cells and NK cells is blocked, which is usually inherited by X-linked recessive way (30). Children with SCID are easy to be infected with various pathogens, most of which are otitis media, pneumonia, and skin infection, as well as disseminated BCG infection. The main clinical feature is that the age of onset is young; most of them are in 27 months. The early clinical manifestations are not typical, which can be manifested as eczema with local delay and refractory, or severe infection, accompanied by long growth retardation. Usually, the clinical manifestations are severe; the infection is challenging to cure, repeated or conditional pathogen infection, persistent diarrhea, dermatitis, and thrush. In this study, 5 years and only 4 months later, severe sepsis and multiple organ failure were the clinical manifestations. Immunoglobulin level, $\mathrm{cd} 3 \mathrm{~cd} 4 \mathrm{~cd} 8 \mathrm{t}$ cells, NK cell count and B lymphocyte count was significantly reduced. IL2RG gene had c. $202 \mathrm{~g}>$ a mutation. The mutation of family sequencing originated from the mother of the child, which can be confirmed as the disease. Also, it has been reported that p. glu68lys mutation was detected in severe combined immunodeficiency (31-33). However, SCID can be caused by multiple gene mutations, so the clinical significance of this mutation is still unclear.

\section{Conclusions}

Although PID has repeated and severe infection, most of them are single-gene genetic diseases, which can be found and treated early. The molecular genetic test of this kind of disease can help children to be diagnosed as early as possible and supply a good quality of life for children through treatment and prevention. In this study, 7 cases of PID diagnosed by gene detection were reported, which enriched the database of PID gene mutation and deepened the understanding of PID by clinicians.

\section{Acknowledgments}

Funding: None.

\section{Footnote}

Conflicts of Interest: All authors have completed the ICMJE uniform disclosure form (available at http://dx.doi. org/10.21037/tp.2020.03.07). The authors have no conflicts of interest to declare.

Ethical Statement: The authors are accountable for all aspects of the work in ensuring that questions related to the accuracy or integrity of any part of the work are appropriately investigated and resolved. The family 
members have approved this study of the children and the medical ethics committee of the hospital (medical ethics approval [2016] NO. 10).

Open Access Statement: This is an Open Access article distributed in accordance with the Creative Commons Attribution-NonCommercial-NoDerivs 4.0 International License (CC BY-NC-ND 4.0), which permits the noncommercial replication and distribution of the article with the strict proviso that no changes or edits are made and the original work is properly cited (including links to both the formal publication through the relevant DOI and the license). See: https://creativecommons.org/licenses/by-nc-nd/4.0/.

\section{References}

1. Kelsen JR, Sullivan KE. Inflammatory bowel disease in primary immunodeficiencies. Curr Allergy Asthma Rep 2017;17:57.

2. De Ranieri D, Sarkoah FN. Intravenous immunoglobulin in the treatment of primary immunodeficiency diseases. Pediatr Ann 2017;46:e8-e12.

3. King JR, Hammarstr ML. Newborn screening for primary immunodeficiency diseases: history, current and future practice. J Clin Immunol 2018;38:56-66.

4. Parvaneh N, Casanova JL, Notarangelo LD, et al. Primary immunodeficiencies: a rapidly evolving story. J Allergy Clin Immunol 2013;131:314-23.

5. Stray-Pedersen A, Sorte HS, Samarakoon P, et al. Primary immunodeficiency diseases: genomic approaches delineate heterogeneous mendelian disorders. J Allergy Clin Immunol 2017;139:232-45.

6. Subspecialty Group of Immunology, the Society of Pediatrics, Chinese Medical Association. Early recognition and screening of primary immunodeficiency diseases. Zhonghua Er Ke Za Zhi 2015;53:893-7.

7. Lawless D, Geier CB, Farmer JR, et al. Prevalence and clinical challenges among adults with primary immunodeficiency and recombination-activating gene deficiency. J Allergy Clin Immunol 2018;141:2303-6.

8. Kohn DB, Kuo CY. New frontiers in the therapy of primaryimmunodeficiency: from gene addition to gene editing. J Allergy Clin Immunol 2017;139:726-32.

9. Modell V, Orange JS, Quinn J, et al. Global report on primary immunodeficiencies: 2018 update from the Jeffrey Modell Centers Network on disease classification, regional trends, treatment modalities, and physician reported outcomes. Immunol Res 2018;66:367-80.
10. Barmettler S, Otani IM, Minhas J, et al. Gastrointestinal manifestations in X-linked agammaglobulinemia. J Clin Immunol 2017;37:287-94.

11. Bush A, Hogg C. The answer is cilia, whatever the question may be! Ann Transl Med 2018;6:S32.

12. Carrillo-Tapia E, García-García E, Herrera-González NE, et al. Delayed diagnosis in $\mathrm{X}$-linked agammaglobulinemia and its relationship to the occurrence of mutations in BTK non-kinase domains. Expert Rev Clin Immunol 2018;14:83-93.

13. Hoshino A, Okuno Y, Migita M, et al. X-linked agammaglobulinemia associated with B-precursor acute lymphoblastic leukemia. J Clin Immunol 2015;35:108-11.

14. Bearden D, Collett M, Quan PL, et al. Enteroviruses in $\mathrm{X}$-linked agammaglobulinemia: update on epidemiology and therapy. J Allergy Clin Immunol Pract 2016;4:1059-65.

15. Giorgetti OB, Paolini MV, Oleastro MM, et al. X-linked agammaglobulinemia in adults clinical evolution. Medicina (B Aires) 2016;76:65-70.

16. Lopez-Herrera G, Berron-Ruiz L, Mogica-Martinez D, et al. Characterization of Bruton's tyrosine kinase mutations in Mexican patients with X-linked agammaglobulinemia. Mol Immunol 2008;45:1094-8.

17. Jin $\mathrm{H}$, Webster $\mathrm{A}$, Vihinen $\mathrm{M}$, et al. Identification of BTK mutations in 20 unrelated patients with X-linked agammaglobuliemia (XLA). Hum Mol Genet 1995;4:693-700.

18. Gaspar HB, Bradley LA, Katz F, et al. Mutation analysis in Bruton's tyrosine kinase, the $\mathrm{X}$-linked agammaglobulinaemia gene, including identification of an insertional hotspot. Hum Mol Genet 1995;4:755-7.

19. Arnold DE, Heimall JR. A Review of Chronic Granulomatous Disease. Adv Ther 2017;34:2543-57.

20. Beaulieu GP, Ward DC, Panch SR, et al. Acanthocytes in the McLeod phenotype of X-linked chronic granulomatous disease. Transfusion 2017;57:2307-8.

21. Labrosse R, Abou-Diab J, Blincoe A, et al. Very EarlyOnset Inflammatory Manifestations of X-Linked Chronic Granulomatous Disease. Front Immunol 2017;8:1167.

22. De Ravin SS, Reik A, Liu PQ, et al. Targeted gene addition in human CD34(+) hematopoietic cells for correction of X-linked chronic granulomatous disease. Nat Biotechnol 2016;34:424-9.

23. Battersby AC, Martin AJ, Tarn J, et al. Raised Serum IL-8 Levels Are Associated with Excessive Fatigue in Female Carriers of X-LinkedChronic Granulomatous Disease in the UK. J Clin Immunol 2017;37:279-81. 
24. Ariga T, Sakiyama Y, Furuta H, et al. Molecular genetic studies of two families with $\mathrm{X}$-linked chronic granulomatous disease: Mutation analysis and definitive determination of carrier status in patients' sisters. Eur J Haematol 1994;52:99-102.

25. Ko SH, Rhim JW, Shin KS, et al. Genetic analysis of CYBB gene in 26 korean families with $\mathrm{X}$-linked chronic granulomatous disease. Immunol Invest 2014;43:585-94.

26. Jhamnani RD, Rosenzweig SD. An update on gain-offunction mutations in primary immunodeficiency diseases. Curr Opin Allergy Clin Immunol 2017;17:391-7.

27. Lorenzini T, Dotta L, Giacomelli M, et al. STAT mutations as program switchers: Turning primary immunodeficiencies into autoimmune diseases. J Leukoc Biol 2017;101:29-38.

28. Takezaki S, Yamada M, Kato M, et al. Chronic mucocutaneous candidiasis caused by a gain-of-function mutation in the STAT1 DNA-binding domain. J Immunol 2012;189:1521-6 .

Cite this article as: Zhu Y, Li L, Mao G, Zhang L, Wang J, Li $\mathrm{N}$. Gene analysis of seven cases of primary immunodeficiency. Transl Pediatr 2020;9(2):117-125. doi: 10.21037/tp.2020.03.07
29. Rivers L, Gaspar HB. Severe combined immunodeficiency: recent developments and guidance on clinical management. Arch Dis Child 2015;100:667-72.

30. Mou W, He J, Chen X, et al. A novel deletion mutation in IL2RG gene results in X-linked severe combined immunodeficiency with an atypical phenotype. Immunogenetics 2017;69:29-38.

31. Markiewicz S, Subtil A, Dautry-Varsat A, et al. Detection of three nonsense mutations and one missense mutation in the interleukin-2 receptor gamma chain gene in SCIDX1 that differently affect the mRNA processing. Genomics 1994;21:291-3.

32. Motobayashi M, Inaba Y, Fukuyama T, et al. Successful treatment for west syndrome with severe combined immunodeficiency. Brain Dev 2015;37:140-4.

33. Yu H, Zhang VW, Stray-Pedersen A, et al. Rapid molecular diagnostics of severe primary immunodeficiency determined by using targeted next-generation sequencing. J Allergy Clin Immunol 2016;138:1142-51.e2. 